\title{
New Views of Solar Energetic Particles From The Advanced Composition Explorer
}

\author{
R. A. Mewaldt \\ California Institute of Technology, Pasadena, CA 91125 USA
}

\begin{abstract}
Since its launch in August 1997, the Advanced Composition Explorer has been measuring the elemental, isotopic, and ionic charge-state composition of solar energetic particles with three new, high-resolution instruments (SEPICA, SIS, and ULEIS). These studies span the energy range from $\sim 20 \mathrm{keV} /$ nucleon to $>100 \mathrm{MeV} /$ nucleon, and include elements from He to $\mathrm{Ni}$ $(2 \leq Z \leq 28)$. This paper reviews early highlights of these investigations and discusses the implications for theories of the origin, acceleration, and transport of solar energetic particles.
\end{abstract}

\section{INTRODUCTION}

The Advanced Composition Explorer (ACE) is presently in orbit about the inner Lagrangian point (L1) carrying new instrumentation designed to measure the composition of energetic nuclei from solar wind energies ( $\sim 1 \mathrm{keV} /$ nucleon) to galactic cosmic ray energies $(\sim 500 \mathrm{MeV} /$ nucleon $)$. A primary goal of $\mathrm{ACE}$ is to compare the composition of material measured in the solar wind and solar energetic particles (SEPs) with that of anomalous and galactic cosmic rays originating from galactic material [1]. These measurements are used to study the origin of these samples of material as well as particle acceleration and transport processes.

ACE includes three new state-of-the-art instruments that together measure the nuclear charge $(Z)$, mass $(M)$, ionic charge state $(Q)$, and kinetic energy $(E)$ of SEP ions from $\mathrm{He}$ to $\mathrm{Ni}(2 \leq \mathrm{Z} \leq 28)$ with significantly improved resolution and collecting power [1]. These include an Ultra Low Energy Ion Spectrometer (ULEIS) that measures $\mathrm{M}$ and $\mathrm{E}$ from $\sim 0.03$ to $\sim 3 \mathrm{MeV} /$ nucleon [2], a Solar Isotope Spectrometer (SIS) that measures $\mathrm{Z}, \mathrm{M}$, and $\mathrm{E}$ from $\sim 10$ to $\sim 100 \mathrm{MeV} /$ nucleon [3], and a Solar Energetic Particle Ionic Charge Analyzer (SEPICA) that determines Q, Z, and E from $\sim 0.2$ to $\sim 1 \mathrm{MeV} /$ nucleon [4]. In addition the Energetic Proton and Alpha Particle Monitor (EPAM) provides measurements of low energy $\mathrm{H}, \mathrm{He}$, and electrons [5]. Data from these and other ACE instruments are now publicly available (see $\mathrm{http} / /$ www.srl.caltech.edu/ACE/), including real-time data from L1 that is available within $\sim 5$ minutes of measurement. This paper summarizes highlights of the new SEP results obtained from $\mathrm{ACE}$ during its first two years.

\section{CLASSES OF SOLAR ENERGETIC PARTICLE EVENTS}

Over the past decade it has been recognized that SEP events fall into two general classes: impulsive and gradual. Although these labels were originally assigned on the

CP516, 26 International Cosmic Ray Conference, edited by B. L. Dingus, et al. (c) 2000 American Institute of Physics 1-56396-939-4/00/\$17.00 
basis of the temporal profiles of the associated x-ray emission, the key function of these categories is to distinguish differences in the acceleration processes involved (see, e.g., $[6,7]$ ). Impulsive SEP events are generally small in size with particle acceleration occurring in association with impulsive solar flares, while gradual events are generally larger events in which particles are accelerated by shocks associated with coronal mass ejections (CMEs; see [8]). Table 1 (based on [6]) summarizes characteristics of these event classes.

TABLE 1. Characteristics of Impulsive and Gradual Solar Particle Events.

\begin{tabular}{lcc}
\hline Characteristic & Impulsive & Gradual \\
\hline${ }^{3} \mathrm{He} /{ }^{4} \mathrm{He}$ & $\sim 1$ & $\sim 0.0004$ \\
$\mathrm{Fe} / \mathrm{O}$ & $\sim 1$ & $\sim 0.1$ \\
$\mathrm{H} / \mathrm{He}$ & $\sim 10$ & $\sim 100$ \\
$\mathrm{Fe}$ Charge State & $\sim 20$ & $\sim 14$ \\
Electron/Proton & Electron-rich & Proton-rich \\
X-rays & Impulsive & Gradual \\
Typical Duration & Hours & Days \\
Longitudinal Cone & $<30^{\circ}$ & $\sim 180^{\circ}$ \\
\hline
\end{tabular}

The ability of ACE to measure composition at very low energies has led to improved understanding of the nature of impulsive events. This is illustrated in Figure 1 , in which the energy/nucleon of individual ions measured by ULEIS is plotted versus time during a five-day period in 1998 [9]. As many as eleven separate SEP events are visible, all showing the effects of time dispersion - higher energy particles arrive first, followed by progressively lower energy ions. These data provide graphic evidence for a picture in which the particles are accelerated impulsively from the same solar active region and all travel the same distance to $\mathrm{L} 1$. The ions in an individual event are highly collimated - in some cases the trace of an event is broken when ACE happens to cross onto adjacent magnetic field lines that are not connected to the acceleration site. Measurements such as these are providing a new tool for studying particle propagation in interplanetary space.

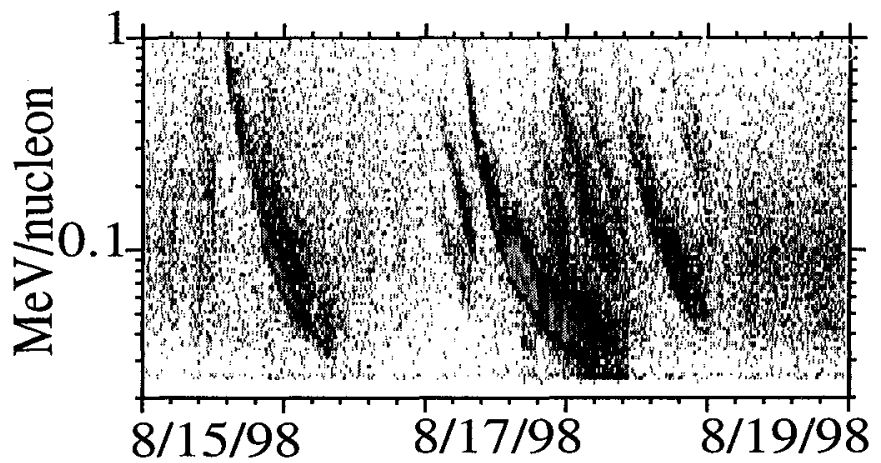

FIGURE 1. Measured kinetic energy versus arrival time for $Z \geq 6$ ions with $>0.02 \mathrm{MeV} /$ nucleon measured by the ULEIS sensor on ACE [9]. Eleven separate SEP events can be recognized by their characteristic shape, which is due to time dispersion effects. Some events are interrupted when the spacecraft moves onto field lines that are not connected to the source region (e.g., early on 8/17). 
One of the intriguing properties of impulsive events is that the rare isotope ${ }^{3} \mathrm{He}$ is often enhanced by factors of $\sim 10^{2}$ to $>10^{4}$ over its abundance in the photosphere and solar wind $\left({ }^{3} \mathrm{He}^{/ 4} \mathrm{He} \sim 0.0004\right)$. These enhancements have been attributed to preferential heating of ${ }^{3} \mathrm{He}$ because of its unique charge-to-mass ratio [10,11]. Studies with the previous generation of instruments have led to a convention of requiring “3 He-rich events" to have ${ }^{3} \mathrm{He} /{ }^{4} \mathrm{He}>0.1$, because smaller ${ }^{3} \mathrm{He}$ enhancements could not be reliably identified [12]. New observations with ULEIS [9] and SIS [13] have revealed many SEP events (including large, presumably gradual events) with ${ }^{3} \mathrm{He} /{ }^{4} \mathrm{He}$ ratios from $\sim 0.001$ to $\sim 0.1$ (see also [14]). Mason et al. [9] suggest that the ${ }^{3} \mathrm{He}$ in gradual events is accelerated from a remnant of small ${ }^{3} \mathrm{He}$-rich events. This remnant forms a seed population for interplanetary shocks that accompany large SEP events.

Figure 2 compares the $\mathrm{Fe} / \mathrm{Mg}$ and ${ }^{3} \mathrm{He} /{ }^{4} \mathrm{He}$ ratios measured by ULEIS in a sample of both large and small SEP events. Previous studies, limited to events with ${ }^{3} \mathrm{He} /{ }^{4} \mathrm{He}>$ 0.1 , had concluded that the magnitudes of the ${ }^{3} \mathrm{He} /{ }^{4} \mathrm{He}$ and heavy ion enhancements in ${ }^{3} \mathrm{He}$-rich events were not correlated [12]. If the correlation indicated in Figure 2 for ratios $>0.02$ is verified by larger samples of SEP events, it will provide important information on the nature of the mechanism that enhances ${ }^{3} \mathrm{He}$.
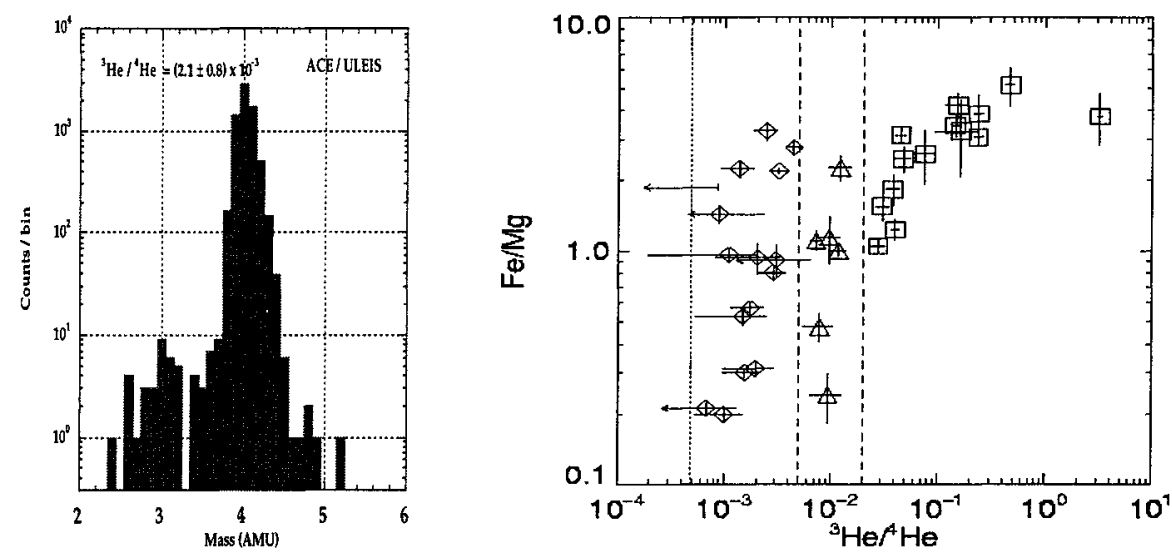

FIGURE 2. (Left) Mass distribution of 0.5 to $2 \mathrm{MeV} /$ nucleon He from the large 11/6/99 event [16]. (Right) Correlation plot of $\mathrm{Fe} / \mathrm{Mg}$ versus ${ }^{3} \mathrm{He} /{ }^{4} \mathrm{He}$ measured in individual SEP events by ULEIS [15].

In addition to the unexpected enhancement of ${ }^{3} \mathrm{He}$ in large SEP events, some of the first measurements from $\mathrm{ACE}$ have shown that distinguishing between the impulsive and gradual classes of events is sometimes difficult [16]. Several of the largest events observed to date have had a composition that is more characteristic of impulsive rather than gradual events [13].

\section{IONIC CHARGE-STATE MEASUREMENTS}

The ionic charge states of SEPs are expected to reflect the temperature of the material from which they were accelerated, as well as subsequent processes that may remove additional electrons. This sensitivity is indicated in Figure 3, where the mean 
charge-to-mass ratios $(\mathrm{Q} / \mathrm{M})$ of several species are shown as a function of temperature, assuming thermal equilibrium conditions. Almost two decades ago measurements on ISEE-3 of $\sim 1 \mathrm{MeV} /$ nucleon ions in sixteen large, gradual-type events showed that the average charge states of elements from $\mathrm{C}$ to $\mathrm{Fe}$ were generally consistent with a temperature of $\sim 2 \mathrm{MK}$, similar to that of the corona [17]. For impulsive events (where it was necessary to sum over $\sim 30$ small events to obtain a large enough data sample) the mean charge state of $\mathrm{Fe}$ was found to be $\sim 20$, characteristic of a temperature of $\sim 10$ MK [18]. The higher characteristic temperature of impulsive events was interpreted as indicating that the ions had been accelerated from flare-heated plasma. The ISEE-3 instrument had sufficient resolution to measure the mean (but not the shape) of the charge-state distributions. It did not provide data on any possible energy dependence of the charge state distributions.

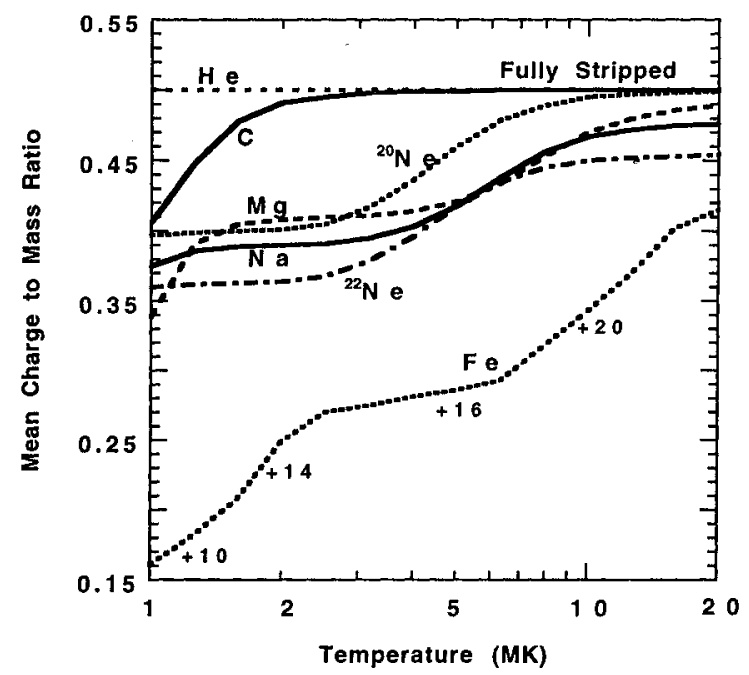

Figure 3. The mean charge-to-mass ratios of several ion species are shown as a function of electron temperatures ranging from $1 \mathrm{MK}$ to $20 \mathrm{MK}$. These curves, which assume thermal equilibrium, are based on calculations by Arnoud and Rothenflug [19] and Arnoud and Raymond [20]. Note that the ionization state of $\mathrm{Fe}$ (indicated below $\mathrm{Fe}$ curve) is expected to be an especially good thermometer.

The SEPICA instrument on ACE is now providing the first direct measurements of SEP charge states since the early 1980 's, with significantly improved resolution and collecting power. Charge-state measurements from the first large SEP event observed by ACE, shown in Figure 4, indicate that the ionization states of several elements increase with increasing energy [21]. At the highest energies measured by SEPICA there is general agreement with the average charge states reported from ISEE-3 [17]. Instruments on the polar-orbiting SAMPEX spacecraft also find an energy dependent ionization state in this event using a geomagnetic technique to measure $\mathrm{Q} / \mathrm{M}[22]$, with an Fe charge state of $\sim 20$ at $>30 \mathrm{MeV} /$ nucleon (see also [13]). Earlier SAMPEX studies of two 1992 events also found that the mean charge-state of Fe increased with energy; in this case the variation was observed above $\sim 10 \mathrm{MeV} /$ nucleon [24].

The interpretation of these energy-dependent charge-state distributions is not yet clear, but initial suggestions center on the time scales for acceleration and ionization 
equilibration, and on the effects of electron stripping (see $[25,26,7])$. It will be important to study additional examples of such events to investigate these possibilities.

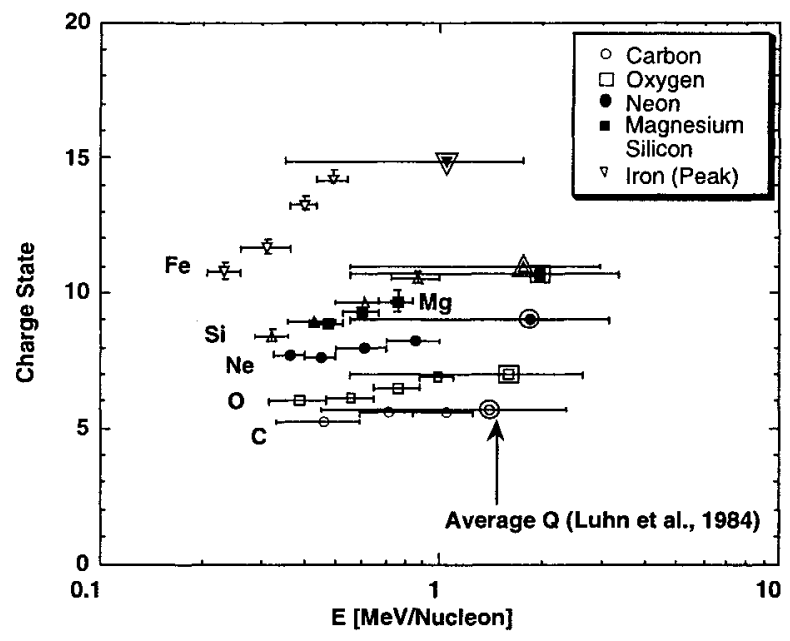

FIGURE 4. SEPICA measurements of ionic charge-states in the 11/6/97 SEP event (from [21]) are compared to the average charge states measured by ISEE-3 [17]. SAMPEX measurements also find energy-dependent charge states in this event using a geomagnetic technique [22].

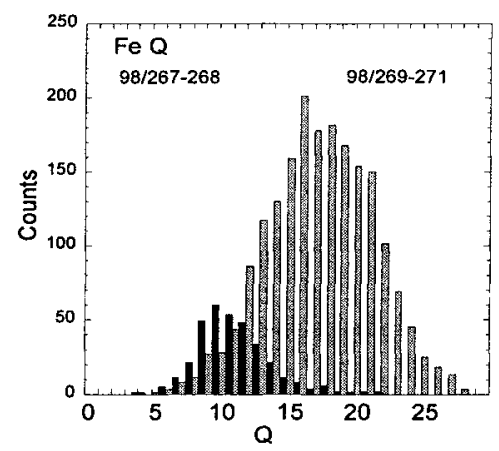

FIGURE 5. Charge state distributions for Fe measured by SEPICA [27] during a small gradual event $($ mean $\approx 10)$ and an impulsive event several days later $($ mean $\approx 17$ ).

SEPICA has also measured charge state distributions in impulsive SEP events and Figure 5 shows two distributions for $\mathrm{Fe}$ measured only days apart, one with a mean of $\sim 10$ from a small gradual event, and one with a mean of $\sim 17$ from an impulsive event. With the improved resolution of SEPICA (rms resolution of $\sim 2$ charge units for $\mathrm{Fe}$ with $<1 \mathrm{MeV} / \mathrm{Q}$ ) it is evident that the $\mathrm{Fe}$ charge-state distributions are often considerably broader than expected from thermal equilibrium, indicating possibly that material from regions of differing temperature was accelerated, or that additional ionization processes have occurred. It is also found that the charge state distributions sometimes evolve with time within a given SEP event [27]. 
Results from many of the first events observed by ACE are summarized in Figure 6 , in which the ionization state of $\mathrm{Fe}$ is plotted versus that of $\mathrm{O}, \mathrm{Ne}$, and $\mathrm{Mg}$ [28]. Also shown are theoretical curves that assume a single equilibrium temperature holds for all species in a given SEP event. While some of the events do appear to satisfy thermal equilibrium, there are also exceptions, including cases where the lighter ions are either more or less ionized than expected. It appears that non-thermal processes may be important in some of these events.

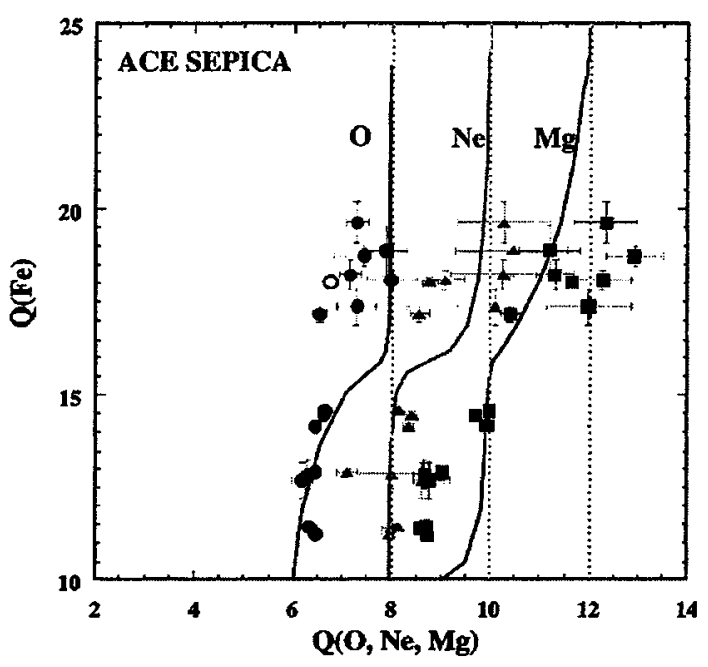

FIGURE 6. Measured charge states of $\mathrm{Fe}$ in a number of different SEP events are plotted versus the measured charge states of $\mathrm{O}, \mathrm{Ne}$, and $\mathrm{Mg}$ [28]. The curves assume thermal equilibrium.

\section{SOLAR ENERGETIC PARTICLE ISOTOPE STUDIES}

Prior to ACE there were only limited measurements of the isotopic composition of ions with $\mathrm{Z} \geq 6$ in solar energetic particles (see, e.g., [29]). ACE has so far observed about a dozen large SEP events in which it was possible to measure the isotopes of a number of heavy elements with the SIS instrument. Examples of the mass resolution achieved are shown in Figure 7. A primary objective of these measurements is to determine the isotopic composition of the corona, since it is very difficult to measure solar isotope abundances using spectroscopic techniques. However, these first measurements have shown that the isotopic composition varies considerably from event to event [30]. For example, the ${ }^{22} \mathrm{Ne}{ }^{20} \mathrm{Ne}$ ratio in the largest events varied by a factor of $\sim 5$ (see also [15]); similar variations are observed for other isotope ratios [31,32]. Although it is well known that the SEP elemental composition varies from event to event, such large variations in the isotopic composition were not expected.

It is natural to ask whether these isotope variations are due to the same acceleration and transport processes that fractionate the elemental composition of SEPs, or whether they result from some additional mass-dependent fractionation process. Breneman 

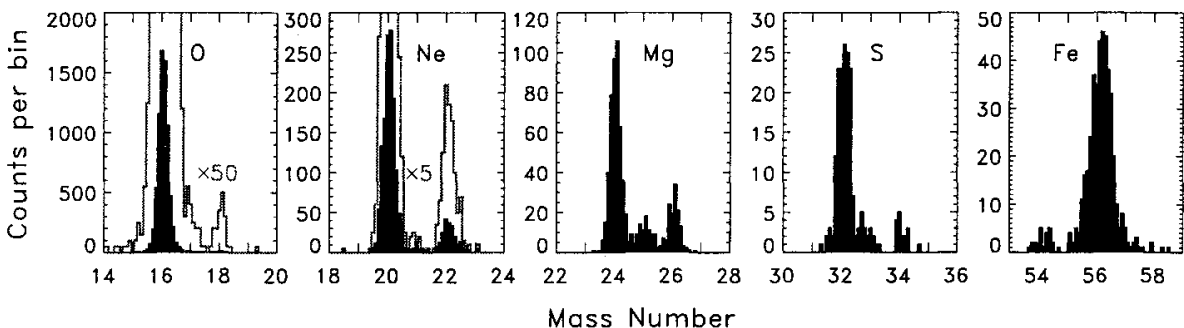

FIGURE 7. Mass distributions of $\mathrm{O}, \mathrm{Ne}, \mathrm{Mg}, \mathrm{S}$, and $\mathrm{Fe}$ ions with $>20 \mathrm{MeV} /$ nucleon measured by the SIS instrument on ACE during the 11/14/98 SEP event [32].

and Stone [33] showed that the ionic charge to mass ratio $(\mathrm{Q} / \mathrm{M})$ is the principal factor for organizing the observed variations in the elemental composition of SEPs. This is possible because the heavier ions (those with $\mathrm{Z} \geq 8$ ) are not fully stripped at typical coronal temperatures of $\sim 1-2 \mathrm{MK}$ (see Figure 3). Thus Fe has a typical Q/M ratio of $\sim 0.25$ at $2 \mathrm{MK}$, while oxygen has a $\mathrm{Q} / \mathrm{M}$ ratio of $\sim 0.45$. Note that for two isotopes of the same element, where the charge states are presumably the same, Q/M-dependent fractionation reduces to mass-dependent fractionation.

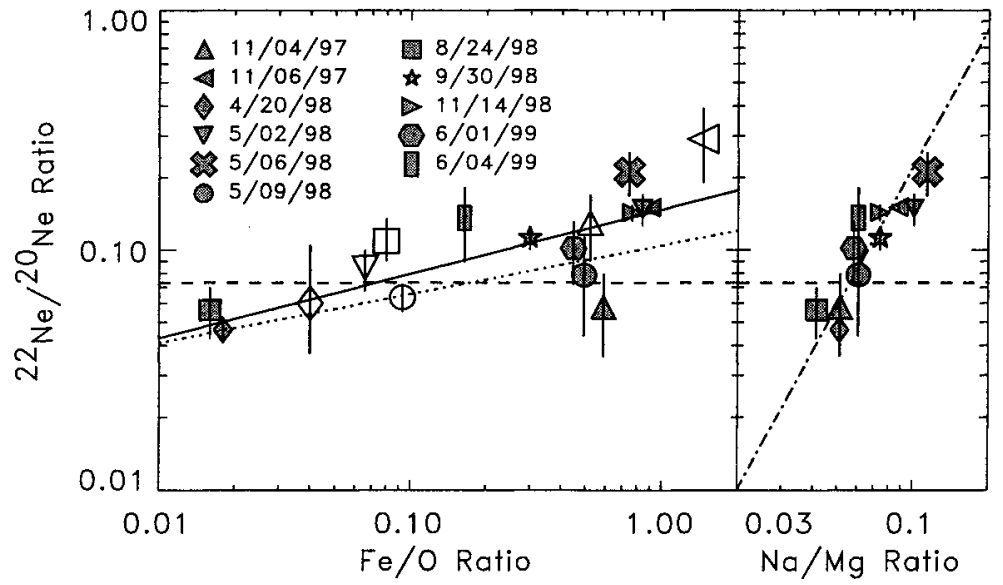

FIGURE 8. Comparison of the ${ }^{22} \mathrm{Ne} /{ }^{20} \mathrm{Ne}$ ratio with the $\mathrm{Fe} / \mathrm{O}$ and $\mathrm{Na} / \mathrm{Mg}$ ratios in SEP events measured by the SIS instrument from $11 / 97$ to $6 / 99$ and with earlier measurements (open symbols summarized in [30]). The curves show possible expected correlations described in $[30,13]$.

Figure 8 shows the correlation between the measured ${ }^{22} \mathrm{Ne} /{ }^{20} \mathrm{Ne}$ ratios and two element ratios, $\mathrm{Fe} / \mathrm{O}$, and $\mathrm{Na} / \mathrm{Mg}$. Note that while there is a strong correlation with $\mathrm{Fe} / \mathrm{O}$, there are also exceptions. This may indicate that there are event-to-event variations in either the $\mathrm{Fe}$ charge state (see above) or the nature of the $\mathrm{Q} / \mathrm{M}$-dependent fractionation. Note that the correlation is much better with the $\mathrm{Na} / \mathrm{Mg}$ ratio, which involves two neighboring elements of Ne. The slope of this correlation can be understood if these ions have charge states typical of $\sim 1.5$ to $4 \mathrm{MK}$ (see Figure 3 and [13]), in which case $\mathrm{Ne}, \mathrm{Na}$, and $\mathrm{Mg}$ are "He-like" ions, each retaining two electrons. 
Bochsler and Kallenbach [34] have offered another possible explanation for the ${ }^{22} \mathrm{Ne} /{ }^{20} \mathrm{Ne}$ variations, pointing out that ${ }^{22} \mathrm{Ne}$ might be enhanced in events where ${ }^{3} \mathrm{He}$ is enriched, since its $\mathrm{Q} / \mathrm{M}$ ratio of $\sim 0.35$ lies closer to the $2^{\text {nd }}$ harmonic of ${ }^{3} \mathrm{He}(1 / 3)$ than does that of ${ }^{20} \mathrm{Ne}(\mathrm{Q} / \mathrm{M}=0.37$; see Figure 3$)$. As discussed above, this model may be relevant to gradual SEP events, since it has been discovered that ${ }^{3} \mathrm{He}$ is also enriched in many gradual events as well as in impulsive SEP events.

The correlation between ${ }^{22} \mathrm{Ne} /{ }^{20} \mathrm{Ne}$ and element ratios such as $\mathrm{Na} / \mathrm{Mg}$ is important because it indicates that by correcting for such variations it will be possible to determine the underlying isotopic composition of coronal material in much the same way as SEP measurements have been used to determine the elemental composition of the corona. Leske et al. [32] have found that variations in other SEP isotope ratios are also correlated with $\mathrm{Na} / \mathrm{Mg}$ and $\mathrm{Fe} / \mathrm{O}$, although the correlation is poorer when it involves species with significantly different $Q / M$ values.

It is interesting that the integrated ${ }^{22} \mathrm{Ne} /{ }^{20} \mathrm{Ne}$ ratio in SEPs during the first 1.5 years of ACE is $\sim 60 \%$ greater than in the solar wind [30], which may be related to a ${ }^{22} \mathrm{Ne}-$ rich component found in lunar soil that has sometimes been attributed to SEPs [35].

\section{SUMMARY}

As is often the case, the first SEP results from ACE have raised more questions than they have answered. Table 1 summarizes some of the ways in which these first results are altering conventional views of solar energetic particle events.

TABLE 2. Summary of New Observations in Solar Particle Events.

\begin{tabular}{ll}
\hline Conventional Picture & New Results \\
\hline $\begin{array}{l}\text { Impulsive: }{ }^{3} \mathrm{He} /{ }^{4} \mathrm{He}>0.1 \\
\text { Gradual: } \quad{ }^{3} \mathrm{He} /{ }^{4} \mathrm{He} \sim 0.0004\end{array}$ & $\begin{array}{l}\text { Impulsive: }{ }^{3} \mathrm{He} /{ }^{4} \mathrm{He}>0.01 \\
\text { Many large events have }{ }^{3} \mathrm{He} /{ }^{4} \mathrm{He} \approx 0.001 \text { to } 0.1\end{array}$ \\
$\begin{array}{l}\text { Impulsive: } \mathrm{Q}(\mathrm{Fe}) \approx 20 \text { at } 1 \mathrm{MeV} / \text { nuc } \\
\text { Gradual: } \mathrm{Q}(\mathrm{Fe}) \approx 15\end{array}$ & $\begin{array}{l}\text { Impulsive: } \mathrm{Q}(\mathrm{Fe}) \approx 17-20 ; \text { broad distribution } \\
\text { Gradual: } \mathrm{Q}(\mathrm{Fe}) \approx 10-15\end{array}$ \\
Charge states assumed independent of energy & Charge states sometimes increase with energy \\
Assume charge states in thermal equilibrium & $\begin{array}{l}\text { Evidence for non-equilibrium ionization } \\
\text { processes }\end{array}$ \\
Elemental composition varies; expect smaller & $\begin{array}{l}\text { Isotope ratios vary by }>\text { x5 from event to event } \\
\text { isotope variations }\end{array}$ \\
Two evtly correlate with elemental composition \\
\end{tabular}

These first results from ACE have come during a period when the Sun was beginning its transition from solar minimum. Solar maximum still lies ahead. As the solar cycle unfolds, it appears that the combination of high-resolution composition data from ACE, complemented by measurements from SAMPEX, Wind, Ulysses, Geotail, SOHO, Yohkoh, and TRACE, will provide an unparalleled opportunity to resolve a number of outstanding questions about solar particle acceleration, as well as providing new insight into the nature of the material that is accelerated. 


\section{ACKNOWLEDGMENTS}

I am grateful to the SEPICA, SIS, and ULEIS teams for providing unpublished data and figures. I especially appreciate discussions with C. Cohen, J. Dwyer, B. Klecker, R. Leske, G. Mason, J. Mazur, E. Moebius, M. Popecki, T. von Rosenvinge, E. Stone, and $\mathrm{M}$. Wiedenbeck, and technical assistance from A. Davis. This research was supported by NASA under grant NAG5-6912.

\section{REFERENCES}

1. Stone, E. C., Frandsen, A. M., Mewaldt, R. A., Christian, E. R., Margolies, D., Ormes, J. F., and Snow, F., Space Sci. Rev. 86, 1-22 (1998).

2. Mason, G. M. et al., Space Sci. Rev. 86, 409-448 (1998).

3. Stone, E. C. et al., Space Sci. Rev, 86, 357-408 (1998).

4. Moebius, E. et al., Space Sci. Rev. 86, 449-496 (1998).

Gold, R. E. et al., Space Sci. Rev. 86, 541-562 (1998).

Reames, D. V., Rev. Geophys. 33, 585-589 (1995).

7. Reames, D. V., this volume.

8. Gosling, J., this volume.

9. Mason, G. M., Mazur, J. E., and Dwyer, J. R., ApJL 525, L133-L136 (1999).

10. Fisk, L. A., ApJ 224, 1048-1055 (1978).

11. Temerin, M., and Roth, I., ApJL 391, L105-L108 (1992).

12. Reames, D.V., Meyer, J.P., and von Rosenvinge, T.T., ApJ Supp. 90, 649-667 (1994).

13. Cohen, C.M.S. et al., Geophys. Res. Lett. 26, 2697-2700 (1999).

14. Chen, J., Guzik, T. G., and Wefel, J. P., ApJ 442, 875 (1995).

15. Dwyer, J. R., Mason, G. M., Mazur, J. E., Gold, R. E., and Krimigis, S. M., Proc. $26^{\text {th }}$ Internat. Cosmic Ray Conf. (Salt Lake City) 6, 147-150 (1999).

16. Mason, G. M. et al., Geophys. Res. Lett. 26, 141-144 (1999).

17. Luhn, A., et al., Proc. 19th Internat. Cosmic Ray Conf. (La Jolla) 4, 241-244 (1985).

18. Luhn, A., Klecker, B., Hovestadt, D., Möbius, E., ApJ 317, 951-955 (1987).

19. Arnaud, M. and Rothenflug, R., Astron. Astrophys. Suppl. Ser. 60, $425-457$ (1985).

20. Arnaud, M. and Raymond, J., ApJ 398, 394-406 (1992).

21. Möbius, E., et al., Geophys. Res. Lett. 26, 145-148 (1999).

22. Mazur, J. E., Mason, G. M., Looper, M. D., Leske, R. A., and Mewaldt, R. A., Geophys. Res. Lett. 26, 173-176 (1999).

23. Cohen, C.M.S. et al., Geophys. Res. Lett. 26, 149-152 (1999).

24. Oetliker, M., et al., ApJ 477, 495-501 (1997).

25. Barghouty, A. F., and Mewaldt, R. A., ApJL 520, L127-L130 (1999).

26. Kartavykh, Yu. Yu., and Ostryakov, V. M., Proc. $26^{\text {th }}$ Internat. Cosmic Ray Conf. (Salt Lake City) 6, 272-275 (1999).

27. Popecki, M. A. et al., Proc. 26 ${ }^{\text {th }}$ Internat. Cosmic Ray Conf. (Salt Lake City) 6, 187-190 (1999).

28. Moebius, E. et al., Proc. $26^{\text {th }}$ Internat. Cosmic Ray Conf. (Salt Lake City) 6, 87-90 (1999).

29. Williams, D. L., Leske, R.A., Mewaldt, R.A., and Stone, E.C., Space Sci. Rev. 85, 379-386 (1998).

30. Leske, R.A., et al., Geophys. Res. Lett. 26, 2693-2696 (1999).

31. Leske, R.A. et al., Geophys. Res. Lett. 26, 153-156 (1999).

32. Leske, R. A. et al., Proc. $26^{\text {th }}$ Internat. Cosmic Ray Conf. (Salt Lake City) 6, 139-142 (1999).

33. Breneman, H. H., and Stone, E. C., ApJL 299, L57-L61 (1985).

34. Bochsler, P., and Kallenbach, R., Meteroritics 29, 653-656 (1994).

35. Weiler, R., Space Sci. Rev. 85, 303-314 (1998). 